\title{
UTILIDAD DIAGNÓSTICA DEL LATIDO POST- EXTRASISTÓLICO EN LA EVALUACIÓN DE RESERVA CONTRÁCTIL MIOCÁRDICA
}

Fernando Riveros MD*, Luis Moya MD.**

\section{Resumen}

El latido postextrasistólico (LPE) como estímulo inotrópico ventricular permitiría identificar zonas isquémicas y por tanto, territorios irrigados por arterias lesionadas que deberían ser intervenidas. El objetivo del presente estudio es determinar las características operativas del LPE para evaluar isquemia miocárdica con respecto a la "prueba de oro" (perfusión miocárdica con isonitrilos).

Pacientes sintomáticos con una prueba de perfusión miocárdica positiva para isquemia, fueron llevados a cateterismo izquierdo. Según el método de Simpson, se comparó en cada segmento el acortamiento radial durante una sístole normal con una posterior a una pausa postextrasistólica. Se registró la presencia o no de un incremento en la contracción y se comparó cada segmento con el estudio no invasivo.

En 140 segmentos miocárdicos se comparó la presencia de hipercontractilidad postextrasistólica (HCPE), se obtuvieron las características operativas de este método diagnóstico y se compararon los resultados con los de la prueba no invasiva. Los datos de sensibilidad (82\%) y especificidad (65\%) del LPE se acercan a los reportados por la "prueba de oro". Se analizan las diferentes características operativas y se concluye que la mayor utilidad del LPE se encuentra al obtener un resultado negativo. La alta sensibilidad del LPE para identificar isquemia miocárdica así como la sencillez para su obtención, nos da una nueva herramienta en la sala de hemodinamia para definir qué arterias deben ser intervenidas en el mismo momento del cateterismo diagnóstico, sin que la prueba de oro pierda su utilidad diagnóstica.

Palabras clave: isquemia miocárdica, perfusión miocárdica con isonitrilos, sensibilidad, especificidad y latido post extrasistólico (no MeSH).

Abreviaturas: latido postextrasistólico (LPE), hipercontractilidad postextrasistólica (HCPE).

\section{Introducción}

La disfunción sistólica de origen isquémico que involucra una región específica del ventrículo izquierdo puede mejorarse al restaurar el flujo sanguíneo, concepto expresado por Rahimtoola ${ }^{1}$ desde 1989 como miocardio hibernante o aturdido. Para valorar la extensión del miocardio isquémico en un territorio del ventrículo izquierdo, se utiliza en la medida que la tecnología lo ha permitido, una gran cantidad de alternativas que incluyen el electrocardiograma con

Fecha recibido: septiembre I de 2006-Fecha aceptado: octubre II de 2006. $*$

* Cardiólogo, residente II de hemodinamia y cardiología intervencionista. Fundación Universitaria de Ciencias de la Salud.

** Jefe del servicio de hemodinamia y cardiología intervencionista. Profesor Titular Fundación Universitaria de Ciencias de la Salud, Hospital de San José, Bogotá D.C, Colombia. cambios durante una prueba de esfuerzo, ${ }^{2,3}$ el estudio de la motilidad segmentaria analizada en el ventriculograma luego de la intervención farmacológica con dobutamina, ${ }^{4,5}$ así como la cuantificación del metabolismo y la perfusión miocárdica analizada mediante tecnología PET (tomografía por emisión de positrones) y SPECT (tomografía computarizada por emisión de fotón único) con $18 \mathrm{~F}$ - fluoro - deoxiglucosa, 201-Talio o 99mTc-sestamibi endovenoso. ${ }^{6}$ En nuestro medio la utilización de medicina nuclear mediante perfusión miocárdica con isonitrilos es uno de los métodos disponibles con una sensibilidad y especificidad del $90 \%$ y $78 \%$ respectivamente ${ }^{7,8}$ y por lo anterior se considera como la prueba de oro. 
La visualización no sólo de la magnitud del movimiento de las paredes sino su velocidad y sincronismo, le da un alto valor diagnóstico al ventriculograma izquierdo. El LPE es un estimulo inotrópico ventricular muy potente el cual causa sobre un área del miocardio que tenga la capacidad residual de contraerse, que lo haga a pesar que se haya visto como una zona acinética o hipocinética en su estado basal. ${ }^{9}$ Esto se manifiesta como un incremento en el acortamiento radial de cada segmento del ventrículo izquierdo que sea isquémico y la ausencia de dicho acortamiento en los segmentos con necrosis establecida. A pesar del conocimiento que se tiene de este concepto, se le da poco valor en la actualidad durante la realización del ventriculograma izquierdo, desperdiciando información que podría ser de gran utilidad, obligando en algunos casos para evaluar isquemia miocárdica a la realización de pruebas no invasivas posteriores al cateterismo diagnóstico, con un aumento de los costos, tiempo hasta la intervención y los riesgos de un segundo cateterismo en caso de requerir cardiología intervencionista. No hay estudios que evalúen la presencia de HCPE con pruebas de viabilidad y/o isquemia miocárdica. Por este motivo desarrollamos este estudio con el objetivo de evaluar la capacidad diagnóstica que tiene el LPE para la evaluación de isquemia miocárdica y comparar sus resultados con los de la perfusión miocárdica (prueba de oro).

\section{Métodos}

Estudio comparativo de pruebas diagnósticas, en el que se incluyeron todos los pacientes sintomáticos con sospecha clínica de enfermedad coronaria activa, remitidos al servicio de hemodinamia de nuestra institución con una prueba de perfusión miocárdica positiva para isquemia significativa (compromiso mayor al $10 \%$ de la masa ventricular). Los pacientes fueron llevados a cateterismo cardíaco izquierdo y coronariografía. Se excluyeron aquellos que tuvieran contraindicación para la realización del ventriculograma izquierdo o que durante la realización del mismo no presentaran extrasístoles ventriculares.

Para efectuar el ventriculograma se utilizó el mismo medio de contraste diatrizoato sódico de meglumina
(Pielograf ${ }^{\circledR}$ ), en dosis de $0.5 \mathrm{ml} / \mathrm{k}$ de peso, utilizando en todos los pacientes la proyección oblicua anterior derecha a $30^{\circ} .{ }^{9}$ Durante la realización del mismo se eligieron los pacientes que presentaron extrasístoles ventriculares; en caso de presentar taquicardia ventricular se escogió para la medición del LPE la última contracción de la salva ventricular. Con el fin de evaluar el porcentaje de acortamiento de cada uno de los segmentos cardíacos, el mismo hemodinamista utilizó la forma descrita por Simpson ${ }^{9}$ para trazar los contornos del ventrículo izquierdo al final de diástole y sístole. El eje longitudinal en diástole se dividió en tres segmentos iguales por líneas perpendiculares en la unión de los segmentos basal con el medio y del medio con el apical, permitiendo diferenciar cinco segmentos: anterobasal, anterolateral, apical, inferior y posterobasal.

De esta manera se obtuvieron cada uno de los radios del ventrículo izquierdo en diástole y en sístole después de un latido normalmente conducido y posterior a una pausa post extrasistólica, obteniendo el acortamiento de cada uno de los segmentos. La longitud de la diferencia entre diástole y sístole de cada uno de los radios dividida por la longitud de cada radio en diástole y multiplicada por 100, permitió obtener el porcentaje de acortamiento de cada radio y la fracción de eyección. Se comparó segmento por segmento y los cálculos fueron realizados en el software del angiógrafo digital TOSHIBA CASS 8000, los resultados se almacenaron en una tabla Excel.

Se definieron las variables de motilidad segmentaria según Pujadas ${ }^{9}$ como: acinesia: porcentaje de acortamiento menor del $5 \%$ en cualquier segmento; hipocinesia: porcentaje de acortamiento entre 6 y $40 \%$ para el segmento anterobasal, 6 y $25 \%$ para los segmentos posterobasal, inferior y anterolateral y entre el $6-15 \%$ para el segmento apical.

Se comparó el porcentaje de acortamiento basal y posterior a una pausa post -extrasistólica en cada uno de los cinco segmentos anotados. Se consideró presente la HPCE si existía un incremento en la contracción de al menos un 5\% del valor inicial. ${ }^{9}$ En caso contrario se consideraba negativo. 


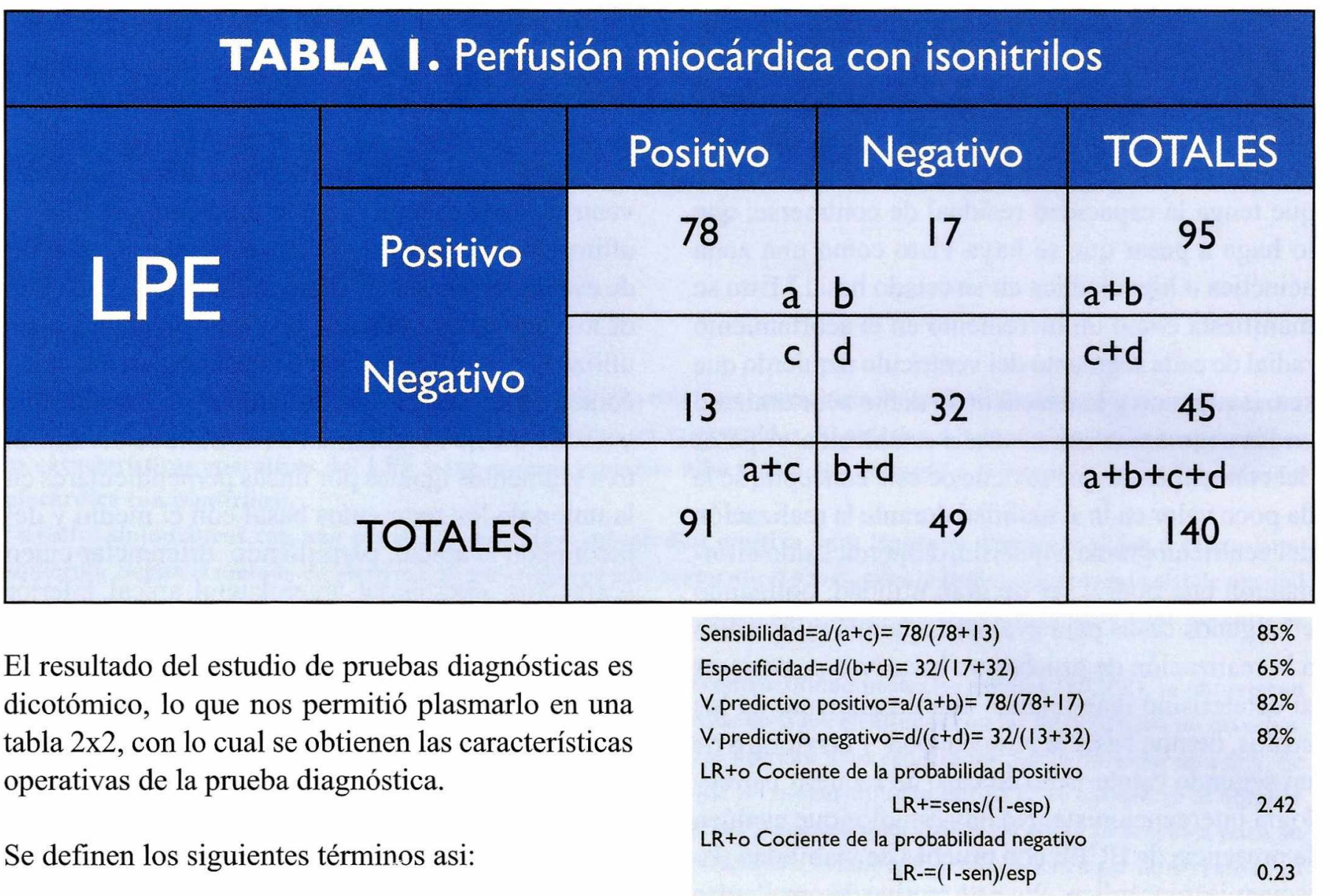

- Sensibilidad: la proporción de verdaderos positivos identificados por la prueba del total de enfermos.

los pacientes antes de la realización del cateterismo cardíaco.

-Especificidad: la proporción de verdaderos negativos identificados por la prueba del total de sanos.

- Valor predictivo positivo: la proporción de sujetos que en verdad tienen la enfermedad, entre los que dieron positivo.

- Valor predictivo negativo: la proporción de sujetos verdaderamente sanos entre el total de los que dieron negativos.

Se estimó para el cálculo del tamaño de muestra (segmentos a evaluar) los siguientes supuestos, alfa de 0.05 , beta de $10 \%$ y un poder de $90 \%$, con una diferencia entre los dos estudios del $20 \%$. El cálculo definitivo fue de 128 segmentos.

Se obtuvo aprobación del comité de ética del hospital y consentimiento informado por parte de todos

\section{Resultados}

Durante un período de seis meses se evaluaron un total de 140 segmentos miocárdicos, en el 100\% se encontró presencia de enfermedad coronaria severa en al menos un vaso (porcentaje de obstrucción mayor o igual al $60 \%$ de la luz arterial). En la Tabla 1 se muestran los resultados de la evaluación de los diferentes segmentos según los resultados de las pruebas y se registran las características operativas del LPE.

El resultado de la sensibilidad del LPE fue de $85 \%$ y la especificidad de $65 \%$. Se obtienen los diferentes índices de valoración de un estudio de pruebas diagnósticas como son el valor predictivo del test positivo y negativo, los cocientes de probabilidad positiva y negativa de la prueba (likelihood ratio). Debido al conocimiento de la probabilidad preprueba 
NOMOGRAMA PARA LA INTERPRETACIÓN DE LOS

RESULTADOS DE LAS PRUEBAS DIAGNÓSTICAS

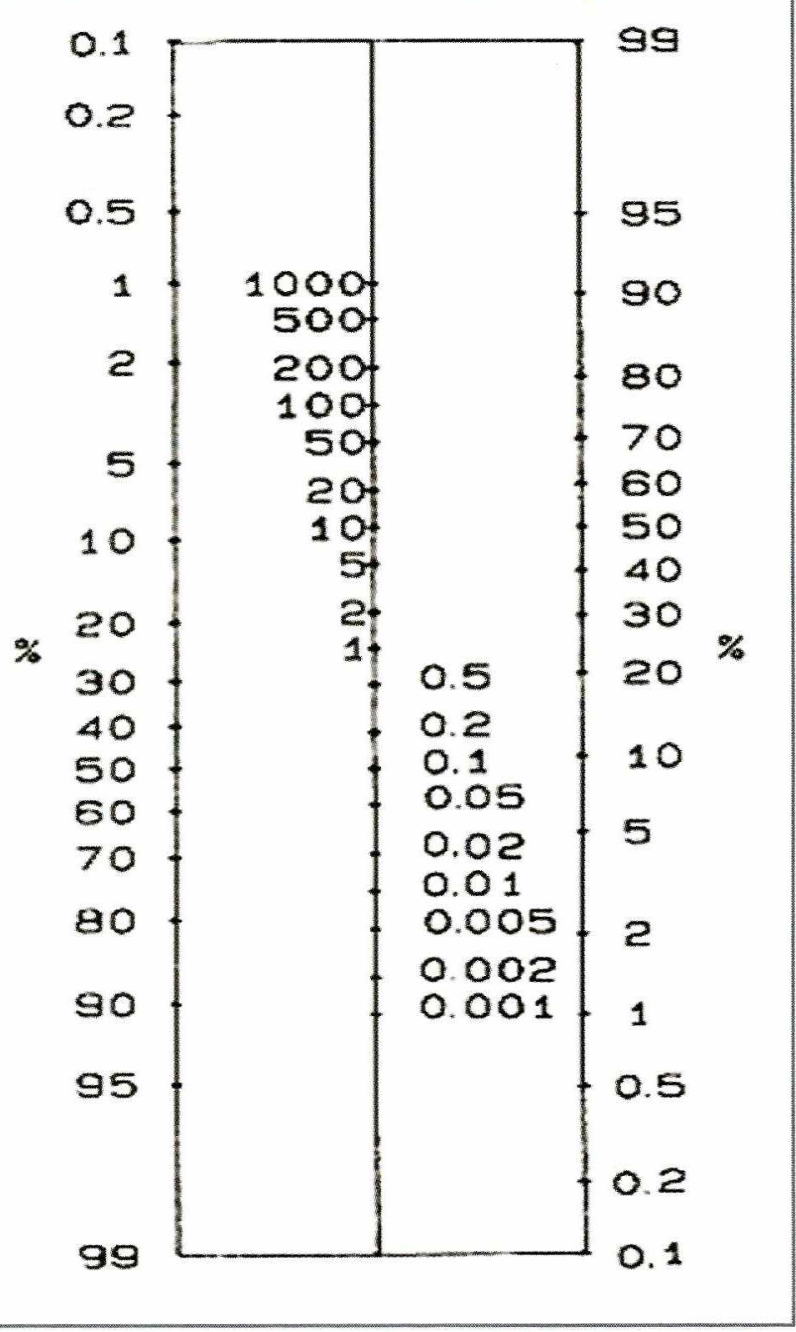

Gráfica I. Nomograma de Fagan para la obtención de la probabilidad postprueba.

y del cociente de probabilidad, se obtiene también mediante el nomograma de Fagan la posibilidad postprueba (Gráfica 1).

\section{Dïscusión}

Aunque fue una interpretación aceptada el hecho de dar valor al LPE, no se tenían estudios comparativos que evaluaran su capacidad diagnóstica con pruebas de isquemia miocárdica. Los resultados obtenidos entre pacientes sintomáticos con enfermedad coronaria confirmada por arteriografía nos muestran que la capacidad diagnóstica del LPE es satisfactoria, ya que los resultados se acercan a los reportados por la prueba de medicina nuclear, con la ventaja adicional de ser un método sencillo de evaluar que no incrementa los costos para el paciente y se puede realizar de manera simultánea con el cateterismo diagnóstico. Aunque la sensibilidad y especificidad evalúan la calidad de la prueba, el interés es responder: ¿Cuál es la probabilidad que tiene un segmento de ser realmente isquémico cuando presenta HCPE? Esta probabilidad se puede calcular debido a que existe la sospecha preprueba de un segmento sea isquémico, teniendo en cuenta que son pacientes sintomáticos con correlación directa por cateterismo de enfermedad coronaria. De esta manera, se obtienen el valor predictivo del test positivo y del test negativo. El uso de estos dos valores en la práctica clínica debe ser cuidadoso, ya que se calculan a partir de la tabla $2 \times 2$ y por lo tanto dependen en forma directa de la proporción de segmentos isquémicos de la muestra estudiada.

Por lo anterior, al determinar los índices de valoración o cocientes de probabilidad positiva y negativa de la prueba (likelihood ratio), los cuales no dependen de la proporción de segmentos enfermos, representan una mejor manera para comparar las dos pruebas.

El hecho de obtener un cociente de probabilidad negativo menor que el de la prueba de oro el cual es de (0.28), indica una mayor capacidad para diagnosticar la ausencia de isquemia miocárdica cuando no hay HCPE. Al evaluar el nomograma de Fagan para la obtención de la probabilidad postprueba, la obtenemos superior al 95\% (Gráfica 1), lo cual nos permite concluir que la posibilidad para un segmento de no tener isquemia residual cuando el resultado del LPE es negativo, es del 95\%. Es de trascendental importancia para la vida de un paciente definir mediante un método diagnóstico de manera precisa la necesidad o no de realizar cardiología intervencionista sobre una arteria determinada. Esta es la fortaleza más importante del estudio. En caso de no documentarse HCPE, se debe considerar que el daño en ese segmento es irreversible y que los esfuerzos por revascularizar la arteria que irriga ese segmento deben ser detenidos. 
Consideramos que en el ventriculograma izquierdo la valoración segmentaria objetiva del acortamiento radial, constituye parte fundamental del cateterismo izquierdo diagnóstico. No se debe evaluar sólo la contracción normalmente conducida, sino que en caso de presentar extrasístoles ventriculares el análisis segmentario posterior a ese estímulo nos brinda información adicional sobre territorios isquémicos.

Por tanto, el ventriculograma tiene otras utilidades y se convierte en herramienta importante para tomar decisiones en la sala de hemodinamia durante la realización del cateterismo cardíaco diagnóstico, sin tener que someter al paciente a una prueba no invasiva posterior para detectar isquemia, ni a un segundo cateterismo para realizar la intervención. Esto se traduce en menor riesgo para el paciente, disminución de tiempo y de los costos para el sistema de salud.

\section{Referencias}

1. Rahimtoola SH. The hibernating myocardium. Am Heart J. 1989; 117:211-21.
2. Mobilia G, Buchberger R. Electrocardiography and myocardial viability. Ital Heart J. 2000; 1(2 Suppl):180-5.

3. Bodi V, Sanchis J. ST segment elevation on Q leads at rest and during exercise: relation with myocardial viability and left ventricular remodeling within the first 6 months after infarction. Am Heart J. 1999; 137(6):1107-15.

4. Shan K, Nagueh. Assessment of myocardial viability with stress echocardiography. Cardiol Clin 1999; 17(3):53953.

5. Haque T, Furukawa T. Myocardial viability detected by dobutamine echocardiography in patients with chronic coronary artery disease, and long term outcome after coronary angioplasty. Jpn Circ J. 2000; 64 (3):183-90.

6. Bergmann SR. Use and limitations of metabolic tracers labeled with positron-emitting radionuclides in the identification of viable myocardium. J Nuc Med. 1994; (suppl):15S-22S.

7. O“Keefe JH, Barnhart CS, Bateman T. Comparation of stress echocardiography and stress myocardial perfusion scintigraphy for diagnosis coronary artery disease and assessing its severity. Am J Cardiol. 1995; 75:25D-34D.

8. Verani MS. Stress myocardial perfusion imaging versus echocardiography for the diagnosis and risk stratification of patients with known or suspected coronary artery disease. Seminars Nucl Med. 1999;4: 319-29.

9. Brenner B. Left ventricular Function. En: Pujadas G. Coronary angiography. 3rd ed. New York: McGraw Hill; 1975.
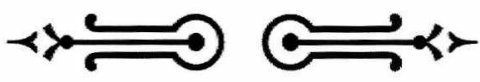